\title{
Lactose digestion in pregnant African-Americans
}

\author{
David M Paige ${ }^{1, *}$, Frank R Witter ${ }^{2}$, Yvonne L Bronner ${ }^{3,4}$, Lisa A Kessler ${ }^{3,5}$, Jay A Perman ${ }^{6,7}$ \\ and Tara R Paige 3,8 \\ 'Department of Population and Family Health Sciences, Johns Hopkins Bloomberg School of Public Health, \\ 624 North Broadway, Room 280, Baltimore, MD 21205 , USA: 2Department of Obstetrics and Gynecology, \\ Johns Hopkins Hospital, Baltimore, MD, USA: ${ }^{3}$ Maternal and Child Health Department, Johns Hopkins School of \\ Hygiene and Public Health, Baltimore, MD, USA: ${ }^{4}$ Current address: Morgan State University, Baltimore, MD, USA: \\ ${ }^{5}$ Current address: $c / o$ Linda Kelly, Johns Hopkins Bloomberg School of Public Health, Baltimore, MD, USA: \\ ${ }^{6}$ Department of Pediatrics, Johns Hopkins University, Baltimore, MD, USA: ${ }^{7}$ Current address: University of Maryland \\ Hospital for Children, Baltimore, MD, USA: ${ }^{8}$ Department of Obstetrics and Gynecology, Underwood Memorial \\ Hospital, Woodbury, NJ, USA
}

Submitted 7 January 2003: Accepted 19 May 2003

\begin{abstract}
Objective: This paper reports on the status of lactose digestion during early and late pregnancy and at 8 weeks postpartum in an African-American population. The hypothesis is that lactose digestion and milk tolerance do not change throughout pregnancy and do not differ from those of non-pregnant African-American women. Design and subjects: This longitudinal study determined lactose digestion after ingesting $240 \mathrm{ml}$ of $1 \%$ fat milk containing $12 \mathrm{~g}$ of lactose at: (1) early pregnancy, prior to 16 weeks ( $n=148)$; (2) late pregnancy, 30-35 weeks $(n=77)$; and (3) 8 weeks postpartum $(n=93)$. One hundred and one comparably matched non-pregnant African-American women served as controls.

Results: Prevalence of lactose digestion, as measured by breath hydrogen, was $80.2 \%$ in the control women, $66.2 \%$ in early pregnancy, $68.8 \%$ in late pregnancy and $75.3 \%$ postpartum. The prevalence of women reporting symptoms was approximately $20 \%$ regardless of lactose absorption status. However, the control women reported significantly more symptoms than did the pregnant women.

Conclusions: This study indicates that there is no significant change in lactose digestion during pregnancy. The prevalence of lactose intolerance for the pregnant African-American women studied is similar to that for non-pregnant African-American women and similar to previous prevalence reports in adult African-Americans. There was no change in the tolerance of lactose noted during pregnancy in these women. There were, however, fewer symptoms reported by the lactose-maldigesting pregnant women.
\end{abstract}

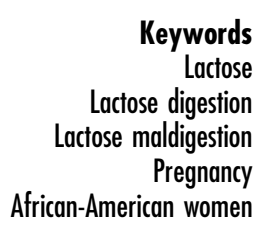

Nutritional reinforcement of pregnant women is an important component of prenatal care. Milk is widely recommended and is strongly emphasised during pregnancy. Milk is provided and encouraged by the United States Department of Agriculture's (USDA) Supplemental Feeding Program for Women, Infants and Children (WIC) directed at low-income, high-risk women and by many governmental and relief agencies throughout the world. The quantity of milk provided to pregnant women participating in the WIC programme may provide approximately half the recommended protein, all of the daily calcium and an energy intake ranging from 17 to $27 \%$ of the daily requirement, depending on the milk selected ${ }^{1}$.

Despite the nutritional value of milk, the lactase levels in some individuals in a number of racial and ethnic groups may be insufficient to hydrolyse commonly consumed amounts of lactose, resulting in lactose maldigestion and possibly milk intolerance ${ }^{2}$. Low lactase levels have been reported in the majority of African-American, Native American, Hispanic, Asian and Indian adults; as well as in $10-15 \%$ of Caucasian adults ${ }^{2}$. While there is a vast literature on lactose digestion, there are only limited data regarding the prevalence of lactose digestion among pregnant African-American women. Despite the unknown status of lactose digestion in pregnant women of AfricanAmerican and other ethnic origin in the United States, milk consumption continues to be encouraged in traditional prenatal nutrition counselling.

The Institute of Medicine (IOM) report on Nutrition During Pregnancy underscores the importance of studying lactose intolerance ${ }^{1}$. The report notes that: 'lactose intolerance among pregnant African-American women may result in their subsequent avoidance of milk'. The IOM report indicates that there are no studies on lactose 
status throughout pregnancy, dietary patterns and milk consumption in pregnant African-American women in the United States. The report further suggests that a decrease in dairy product consumption may result from the higher prevalence of lactose intolerance among African-Americans, and may explain the avoidance of dairy products by pregnant African-Americans participating in the USDA's WIC programme for pregnant women, as reported by Rush et al. $^{3}$.

Despite the publication of a vast scientific literature on the subject of lactose maldigestion and milk intolerance ${ }^{2}$, there are few published studies on lactose digestion during pregnancy. One, an early study from our group in 1973, does examine lactose status in both AfricanAmerican and white pregnant populations ${ }^{4}$. The prevalence of lactose maldigestion as evidenced by an inadequate blood sugar rise was reported as $9 \%$ in the white women and 66\% in the African-American women. This study reported that pregnancy did not alter lactose digestion ${ }^{4}$. A similar profile was reported in Asian Indian communities by Tandon et al. in $1977^{5}$.

Another study conducted with pregnant women was published in 1988 on a population of Guatemalan women ${ }^{6}$. Villar et al. suggested a progressive adaptation to lactose during pregnancy. The investigators found that, as pregnancy progressed, absorption of $18 \mathrm{~g}$ of lactose in $360 \mathrm{ml}$ of milk improved between the early and late period of pregnancy, as judged by breath hydrogen response. Lactose maldigestion among 114 pregnant women tested before the 15th week of gestation was 54\%; breath hydrogen was 116.6 (standard error ( $\mathrm{SE} \pm 9.6$ ) ppm. By term, $44 \%$ of those originally classified as maldigesters were reported to be digesters; breath hydrogen was 54.4 $(\mathrm{SE} \pm 7.3) \mathrm{ppm}(P<0.07)$. The authors interpreted this lower, but still increased value over baseline level in breath hydrogen as an apparent adaptive improvement. Villar's group used a study time of $4 \mathrm{~h}$ to observe a hydrogen breath rise due to increased transit time during pregnancy ${ }^{6}$. Szilagyi et al. reported in 1996 that 16 Canadian women with lactose malabsorption were able to handle lactose better than usual in late pregnancy ${ }^{7}$.

Therefore, the objectives of the present study were (1) to provide data on the prevalence of lactose maldigestion and intolerance in a pregnant African-American population, as determined by breath hydrogen excretion over a period of $8 \mathrm{~h}$; and (2) to determine the changes, if any, in lactose digestion throughout pregnancy and the postpartum period.

\section{Subjects and methods}

Study subjects and controls were recruited from one of four obstetric/gynaecological clinics that are part of the Johns Hopkins Hospital system. All African-American women prior to 16 weeks of pregnancy and meeting study criteria were eligible to participate. Study criteria included:
(1) no record of overt intestinal disease, (2) no history of diabetes or other metabolic disorder, (3) no obstetric complications and (4) eligible for enrolment in the WIC programme. The control women were selected randomly from the family planning centres operating at the same clinics; however, they were not pregnant, nor had they been pregnant within the past three months, as well as having no record of intestinal disease or history of diabetes. The control women were studied independently at a single point in time. The subjects were studied during early pregnancy (13-16 weeks), late pregnancy (30-35 weeks) and at 8 weeks postpartum. The cohort of pregnant women was compared to controls, as well as to themselves over time. The final sample of subjects and control groups indicates sufficient power (0.90) to detect a statistically significant difference in the proportion of women changing lactose status (0.20) and a difference of one-half standard deviation in mean peak hydrogen rise. Informed consent was obtained from all subjects and controls. The protocol was approved by the Johns Hopkins Committee on Human Volunteers.

At the time of recruitment, all study and control women were advised in detail of the study protocol and given an appointment for the breath hydrogen study to determine lactose status. A written notice and telephone reminder call were given. All women were instructed to fast after $20.00 \mathrm{~h}$ the night before testing. A standardised pre-test dinner was not prescribed. They were permitted to have only water. They reported to the hospital testing room at $08.30 \mathrm{~h}$ in the morning. They were greeted by study personnel and re-evaluated as to current health status and ability to meet study criteria. Women who were currently taking antibiotics or had been taking them in the previous 10 days were rescheduled to a later time, such that at least 10 days would have passed since taking the medication. This affected three women.

The women provided an initial breath sample to establish a baseline breath hydrogen value. The study coordinator provided to the subject and observed her drink $240 \mathrm{ml}$ of low-fat (1\%) milk containing $12 \mathrm{~g}$ of lactose. To minimise any difference in milk composition and lactose content, all milk products were obtained from the same source. Subjects did not eat or drink anything for the next $8 \mathrm{~h}$. Breath samples were collected from subjects every $30 \mathrm{~min}$ thereafter by the nasal prong technique, which provides an end-alveolar air sample ${ }^{8}$. At each collection, a nasal prong was placed in the subject's anterior nares by herself or by the study co-ordinator. While breathing normally the subject aspirated repeatedly, into a $60 \mathrm{ml}$ plastic syringe equipped with a one-way stopcock, a $3.5 \mathrm{~cm}^{3}$ fraction of each tidal volume late in the expiratory phase until sufficient volume (approximately $30-50 \mathrm{~cm}^{3}$ ) was obtained. Several times a day, the laboratory coordinator collected the samples for immediate analysis. This format allowed for continuous monitoring of and feedback on the testing procedure. Hydrogen 
concentration was measured by gas chromatography using a Quintron DP Microlyzer?. Concentrations of hydrogen in samples collected by nasal prong showed a consistent relationship with mean alveolar hydrogen, based on comparison with samples obtained by HaldanePriestly tube. The classic Haldane-Priestly method, which secures an end expiratory specimen, has been demonstrated to approximate alveolar gas composition. In addition to breath hydrogen analysis, carbon dioxide concentration was measured as an internal standard for quality control and to normalise hydrogen values in individual samples. Carbon dioxide concentration was measured using a Beckman LB-3 $\mathrm{CO}_{2}$ monitor.

The $\mathrm{CO}_{2}$ monitor was calibrated with a standard blood gas mixture of $5 \% \mathrm{CO}_{2}$, balance $\mathrm{N}_{2}$. $\mathrm{CO}_{2}$ was measured in $\mathrm{mmHg}$. The Quintron DP Microlyzer was calibrated with a primary standard gas mixture of $62.4 \mathrm{ppm} \mathrm{H}_{2}, 22 \mathrm{ppm} \mathrm{CH}_{4}$, $5 \% \mathrm{CO}_{2}$ and balance of air. Once a week the microlyzer was checked for linearity using different concentrations of the standard gas. Samples were analysed for $\mathrm{CO}_{2} \mathrm{H}_{2}$ and $\mathrm{CH}_{4}$ concentrations. The $\mathrm{H}_{2}$ value was corrected to an alveolar concentration using the $\mathrm{CO}_{2}$ concentration.

Women who failed to demonstrate a breath hydrogen rise following the ingestion of lactose returned to be tested to determine their capacity to produce hydrogen. Lactulose, a non-absorbable disaccharide, was used to determine their status. Of the 27 women who were tested with lactulose, one woman was unable to produce hydrogen. Her test results were eliminated from the analysis. The plan of analysis provided for exploration of each of the results of interest in the early and late stages of pregnancy, the postpartum period and in the controls. Pairwise differences were analysed utilising $t$-tests and chisquare tests for correlated proportion. Data were further explored using the Cochran $Q$-test, Mantel-Haenszel and likelihood ratio tests, and reported as $\chi^{2}$ values.

Subjects were given a form to record the occurrence of flatulence, diarrhoea, abdominal cramps and bloating during the $8 \mathrm{~h}$. The symptoms were rated on a scale as none, slight, moderate and severe.

The subjects were in a spacious and comfortable room. During the morning hours, they were interviewed regarding their dietary practices. During the afternoon, they watched videos, read magazines and played card games. After the $8 \mathrm{~h}$ of breath testing, the subjects were reimbursed for their time and travel and received a complimentary meal from the hospital cafeteria. The women were monitored by the study personnel continuously to provide support, answer any questions, identify anyone who evidenced lactose-induced symptoms and to consult with the study obstetrician as needed.

\section{Results}

A total of 274 pregnant subjects were recruited into the study. Ninety-one (33\%) failed to appear twice for their appointments, despite reminder calls. Seven (2.5\%) refused to participate in the breath hydrogen testing after they arrived in the morning. Thirteen (4.7\%) were no longer pregnant at the time of the study appointment and 15 (5.5\%) were deemed ineligible ( $>16$ weeks' pregnancy, on antibiotics or above 35 years of age). One hundred and forty-eight women completed the initial early pregnancy lactose testing.

During late pregnancy, 77 women (52\%) completed the breath hydrogen testing. Thirty-seven (25\%) of the original 148 women had an early termination of their pregnancy (18 miscarriages, three still births and 16 pre-term (26-34 weeks' gestation) deliveries). An additional 34 (23\%) of the original 148 women initially studied were not restudied. One woman moved out of state. Five women refused to continue past the first testing, stating reasons such as: not being able to take time off from work or being unable to wake up early enough to take the test. The remaining 28 (19\%) of the pregnant subjects were lost to follow-up.

Ninety-three women (63\%) completed the 8 weeks postpartum testing. All 77 women studied in late pregnancy were restudied during the postpartum period. In addition, all 16 women with pre-term deliveries who were unable to be studied during late pregnancy were restudied during the postpartum period. There were no statistically significant differences in the demographic characteristics or the lactose status of those women for whom an initial test was conducted, but who did not complete the study because of either loss to follow-up or loss of foetus.

There were 157 women recruited as controls. Forty-nine (31\%) failed to appear twice for their appointment, despite numerous reminder telephone calls. Four (2.5\%) were deemed ineligible because they were older than 35 years. Three $(1.9 \%)$ refused to participate in the testing, after coming in the morning. In total, 101 control women completed the breath hydrogen testing. However, two failed to turn in their report of symptoms.

Demographic characteristics of the subjects and controls are provided in Table 1 . The control group was older. They had a higher number of previous live births and a higher level of education.

\section{Lactose status}

Lactose maldigestion was defined as a rise of more than $20 \mathrm{ppm}$ in breath hydrogen. An increase of $>20 \mathrm{ppm}$ hydrogen above baseline has been accepted as a positive

Table 1 Demographic characteristics (mean (standard deviation)) of African-American study and control women

\begin{tabular}{lccc}
\hline Variable & $\begin{array}{c}\text { Subjects } \\
(n=148)\end{array}$ & $\begin{array}{c}\text { Controls } \\
(n=101)\end{array}$ & $P$-value \\
\hline Age (years) & $21.9(6.0)$ & $27.2(7.5)$ & $<0.001^{*}$ \\
Education (years completed) & $10.8(1.46)$ & $11.2(1.52)$ & $0.02^{*}$ \\
Number of previous pregnancies & $0.68(0.47)$ & $0.77(0.43)$ & 0.14 \\
Number of previous live births & $0.51(0.50)$ & $0.77(0.43)$ & $<0.001^{*}$ \\
\hline
\end{tabular}

${ }^{*}$ By $t$-test. 
indicator of lactose maldigestion ${ }^{10}$. The proportion of nonpregnant control women characterised as lactose-maldigesting was $80.2 \%$. The prevalence of subjects identified as lactose-maldigesting by breath hydrogen analysis at each time period studied is shown in Table 2 . A probability value of $<0.05$ was considered significant. When using a continuity-adjusted chi-square analysis, there was no significant change in the prevalence of lactose malabsorption from early pregnancy to late pregnancy to 8 weeks postpartum. However, there was a significant difference $(P=0.026)$ between the prevalence of lactose maldigestion for subjects during early pregnancy (66.2\% maldigesters) compared with the control women (80.2\%). The breath hydrogen concentrations of the same subjects studied at 13-16 weeks and 30-35 weeks of pregnancy and again at 8 weeks postpartum and control women are depicted in Fig. 1. Lactose-maldigesting pregnant subjects experienced their peak breath hydrogen value at the fifth hour of testing, both early and late in the pregnancy. All of the lactose-maldigesting subjects were still exhibiting a rise in breath hydrogen at the seventh hour of the test. At

Table 2 Prevalence of lactose maldigestion during early and late pregnancy and the postpartum period, and in non-pregnant control women, as determined by breath hydrogen rise of $>20 \mathrm{ppm}$

\begin{tabular}{lcc}
\hline Study period & $n$ & $\begin{array}{c}\text { Prevalence } \\
\text { of lactose } \\
\text { maldigestion } \\
(\%)\end{array}$ \\
\hline Early pregnancy (13-16 weeks) & 148 & $66.2^{*}$ \\
Late pregnancy (30-35 weeks) & 77 & 68.8 \\
8 weeks postpartum & 93 & 75.3 \\
Non-pregnant control women & 101 & 80.2 \\
\hline
\end{tabular}

${ }^{*}$ Continuity-adjusted chi-square test, $P=0.026$ compared with the control women.

There is no significant change in the prevalence of lactose maldigestion between pregnancy stages. the 8 weeks postpartum test the same women evidenced a peak breath hydrogen rise at the fourth hour, paralleling the lactose-maldigesting non-pregnant control pattern. Table 3 lists breath hydrogen values at baseline and at each of the eight hours of the study for all women, early and late in pregnancy, postpartum and controls, by lactose digestion status.

\section{Symptoms}

Subjects and controls were asked to record their symptoms as none, slight, moderate or severe. The milk product was not identified. Subjects and controls expressed difficulty differentiating among levels of symptoms (slight vs. moderate vs. severe). Given the subjective nature of the grading system and lack of interrater reliability, symptoms were analysed as present or absent. Table 4 reports symptoms for each stage of pregnancy for study subjects compared with controls. As determined by chi-square analysis, subjects in the early stages of pregnancy reported significantly less flatulence and diarrhoea than did the control women after the ingestion of $240 \mathrm{ml}$ of low-fat (1\%) milk. Flatulence in early pregnancy was reported in $21.5 \%$ and diarrhoea in only $1.4 \%$, compared with $37.4 \%$ and $11.1 \%$, respectively, in control women $(P<0.009)$. This pattern continued during the later period of pregnancy and postpartum. Subjects reported significantly less flatulence $(21.1 \%$ and $22.3 \%$ vs. $37.4 \%)$, diarrhoea ( $1.3 \%$ and $2.1 \%$ vs. $11.1 \%)$ and bloating $(9.2 \%$ and $8.5 \%$ vs. $23.5 \%)$ than the control women $(P<0.025$ or $<0.009)$.

Regardless of the lactose digestion status of study subjects, they reported a similar presence of symptoms after the ingestion of $240 \mathrm{ml}$ of low-fat (1\%) milk. Flatulence was the only symptom reported at a statistically significant higher level $(P<0.025)$ in the lactose-digesting controls (Table 5).
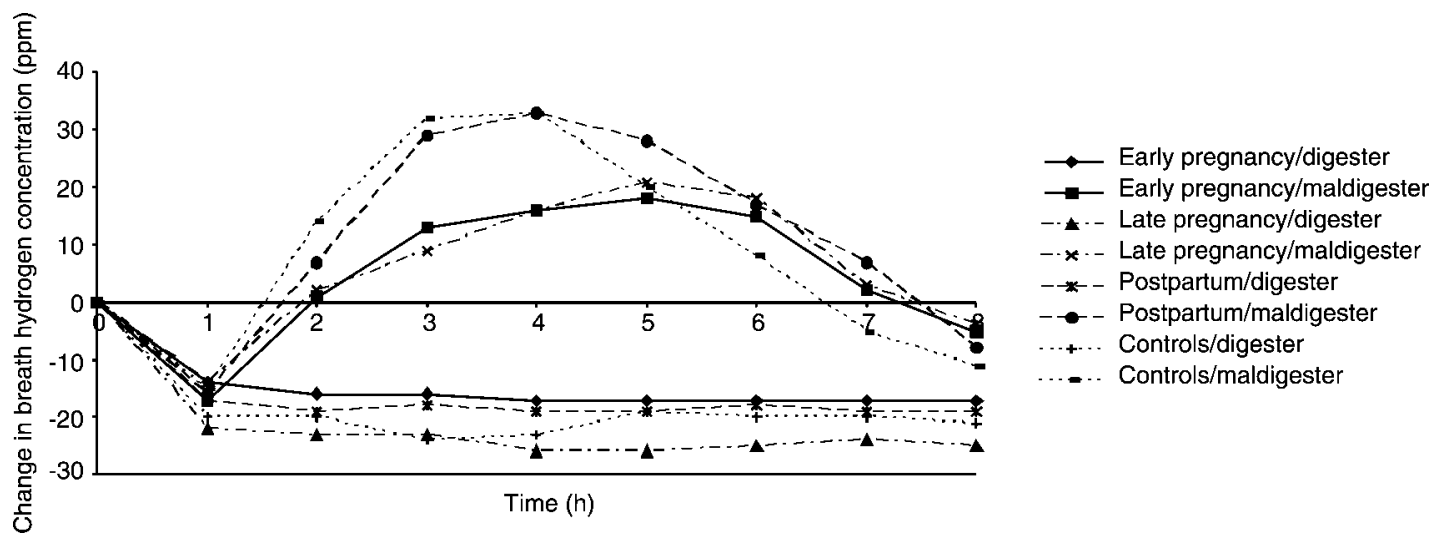
Table 3 Breath hydrogen value (standard error) (ppm) at baseline and at each study hour for study subjects during early and late pregnancy and the postpartum period, and in non-pregnant control women. Reported for all women, lactose maldigesters and lactose digesters

\begin{tabular}{|c|c|c|c|c|}
\hline & Early pregnancy & Late pregnancy & Postpartum & Controls \\
\hline All women & $n=148$ & $n=77$ & $n=93$ & $n=101$ \\
\hline Baseline & $14.75(1.12)$ & $15.97(1.34)$ & $18.17(1.52)$ & $16.76(1.78)$ \\
\hline Hour 1 & $-1.99(0.64)$ & $0.96(0.93)$ & 1.79 (1.29) & $0.30(1.33)$ \\
\hline 2 & $10.05(3.06)$ & $10.66(3.70)$ & $18.13(4.16)$ & $24.5(4.00)$ \\
\hline 3 & $17.74(3.17)$ & 15.07 (3.56) & $34.96(5.66)$ & $37.2(4.61)$ \\
\hline 4 & 19.66 (3.05) & $18.79(4.02)$ & $37.43(5.54)$ & 38.7 (5.03) \\
\hline 5 & $21.21(2.83)$ & 22.42 (3.97) & 34.24 (4.57) & $29.1(3.95)$ \\
\hline 6 & $19.24(3.38)$ & $20.33(3.58)$ & $26.21(4.39)$ & $19.4(2.98)$ \\
\hline 7 & $10.28(1.96)$ & $10.33(2.49)$ & $19.05(3.50)$ & $8.93(2.17)$ \\
\hline 8 & $6.07(1.83)$ & $5.66(2.11)$ & $7.24(2.29)$ & $3.39(2.08)$ \\
\hline Maldigesters & $n=97$ & $n=53$ & $n=70$ & $n=81$ \\
\hline Baseline & $15.59(1.31)$ & $14.28(1.65)$ & $18.86(1.84)$ & $16.44(1.96)$ \\
\hline Hour 1 & $-2.33(0.92)$ & $-0.29(1.23)$ & $2.77(1.59)$ & $1.12(1.66)$ \\
\hline 2 & $16.56(4.39)$ & $16.19(5.22)$ & $25.41(5.28)$ & $30.78(4.66)$ \\
\hline 3 & $28.84(4.31)$ & 23.79 (4.73) & $47.13(6.85)$ & $48.36(5.05)$ \\
\hline 4 & 31.81 (3.94) & $30.88(5.09)$ & $51.85(6.56)$ & $49.95(5.61)$ \\
\hline 5 & $33.71(3.45)$ & $35.94(4.75)$ & $46.46(6.20)$ & $36.82(4.47)$ \\
\hline 6 & $30.74(4.45)$ & $32.77(4.23)$ & $35.44(5.26)$ & 24.85 (3.40) \\
\hline 7 & $17.55(2.42)$ & $17.56(3.10)$ & $25.95(4.14)$ & $11.77(2.55)$ \\
\hline 8 & $10.10(2.37)$ & $10.38(2.66)$ & $10.86(2.80)$ & $5.12(2.49)$ \\
\hline Digesters & $n=50$ & $n=24$ & $n=23$ & $n=20$ \\
\hline Baseline & $13.11(2.07)$ & $20.38(2.22)$ & $16.07(2.58)$ & 18.08 (4.25) \\
\hline Hour 1 & $-1.32(0.12)$ & $-2.73(1.37)$ & $-1.07(1.95)$ & $-2.83(2.43)$ \\
\hline 2 & $-3.10(1.29)$ & $-3.25(1.56)$ & $-3.07(1.76)$ & $-2.13(1.85)$ \\
\hline 3 & $-3.54(1.58)$ & $-3.75(1.82)$ & $-2.55(2.17)$ & $-6.03(2.29)$ \\
\hline 4 & $-4.63(1.85)$ & $-6.58(2.02)$ & $-3.93(2.34)$ & $-5.43(3.37)$ \\
\hline 5 & $-4.05(2.10)$ & $-6.54(1.91)$ & $-3.50(2.25)$ & $-1.97(2.69)$ \\
\hline 6 & $-4.48(2.19)$ & $-6.75(2.24)$ & $-2.81(2.17)$ & $2.97(1.75)$ \\
\hline 7 & $-4.28(2.17)$ & $-4.96(2.15)$ & $-3.73(2.51)$ & $-2.87(2.13)$ \\
\hline 8 & $-4.97(2.14)$ & $-5.63(2.04)$ & $-3.95(2.26)$ & $-3.82(2.23)$ \\
\hline
\end{tabular}

\section{Discussion}

Lactose digestion status, as determined by breath hydrogen rise, did not change significantly throughout pregnancy and the postpartum period. Peak breath hydrogen rise occurred at the fifth to sixth hour of the test in study women both early and late in pregnancy and at the fourth hour in the same women at 8 weeks postpartum. Peak breath hydrogen rise was observed at the fourth hour in the non-pregnant lactose-maldigesting control women. The pregnancy pattern was again reported in the postpartum period, with similar prevalence values for flatulence, diarrhoea and bloating remaining significantly $(P<0.025)$ lower than in control women.

Table 4 Reported symptoms (\%) during early and late pregnancy and the postpartum period, and in non-pregnant control women, after ingestion of $240 \mathrm{ml}$ of low-fat (1\%) milk containing $12 \mathrm{~g}$ of lactose

\begin{tabular}{lcccc}
\hline Symptom & $\begin{array}{c}\text { Early pregnancy } \\
(n=148)\end{array}$ & $\begin{array}{c}\text { Late pregnancy } \\
(n=77)\end{array}$ & $\begin{array}{c}\text { Postpartum } \\
(n=93)\end{array}$ & $\begin{array}{c}\text { Controls } \\
(n=99)\end{array}$ \\
\hline Flatulence & $21.5^{\star *}$ & $21.1^{*}$ & $22.3^{*}$ & 37.4 \\
Diarrhoea & $1.4^{* *}$ & $1.3^{*}$ & $2.1^{\star}$ & 11.1 \\
Abdominal & 24.3 & 18.4 & 13.8 & 29.6 \\
pain & 17.4 & $9.2^{*}$ & $8.5^{* *}$ & 23.5 \\
Bloating & 17.4 & & & \\
\hline
\end{tabular}

${ }^{*}, P<0.025 ;{ }^{* *}, P<0.009$.
Our study indicates that many of the pregnant women have their peak hydrogen rise after $4 \mathrm{~h}$. Therefore, limiting breath hydrogen testing to $4 \mathrm{~h}$ as reported by Villar et al. may misclassify women as lactose digesters. Alternatively, pregnancy may cause blunting of the hydrogen curve that can be due to a change in transit time, adaptation or as yet unrecognised factors. Nevertheless, the prevalence rates of lactose maldigestion in pregnant subjects and nonpregnant controls were similar to those found in previous reports of African-American women ${ }^{4,11}$.

When comparing the symptoms reported by subjects in the early period of pregnancy with the controls, subjects reported significantly less flatulence and diarrhoea. In late pregnancy and at 8 weeks postpartum, the subjects continued to report significantly fewer symptoms (flatulence, diarrhoea and bloating) than the control women. One possible explanation for this inconsistency is that the increased transit time during pregnancy ${ }^{12,13}$ decreases the amount of lactose coming into contact with the jejunal mucosa of the small intestine at any one time and therefore minimises discomfort. Another possible explanation for the report of fewer symptoms by the pregnant women is that they may have become less sensitive to intestinal discomfort and are less inclined to report symptoms.

Approximately $20 \%$ of the women, regardless of lactose digestion status, reported symptoms of milk intolerance (bloating, diarrhoea, flatulence and/or abdominal pain). 
Table 5 Reported symptoms (\%) during early and late pregnancy and the postpartum period, and in non-pregnant control women, after ingestion of $240 \mathrm{ml}$ of low-fat (1\%) milk containing $12 \mathrm{~g}$ of lactose, by lactose status

\begin{tabular}{lcccc}
\hline Symptom & Early pregnancy & Late pregnancy & Postpartum & Controls \\
\hline Digesters & $n=50$ & $n=24$ & $n=23$ & $n=19$ \\
Flatulence & 12.2 & 20.8 & 8.7 & 15.0 \\
Diarrhoea & 2.0 & 0.0 & 0.0 & 5.0 \\
Abdominal pain & 16.3 & 25.0 & 17.4 & 20.0 \\
Bloating & 16.3 & 12.5 & 8.7 & 25.0 \\
Maldigesters & $n=98$ & $n=53$ & $n=70$ & $n=80$ \\
Flatulence & 25.5 & 20.8 & 27.1 & $43.2^{*}$ \\
Diarrhoea & 0.0 & 1.9 & 2.9 & 13.6 \\
Abdominal pain & 27.7 & 17.0 & 12.9 & 32.1 \\
Bloating & 19.2 & 7.6 & 7.1 & 23.5 \\
\hline
\end{tabular}

${ }^{*}, P<0.025$.

This finding is similar to that found by Newcomer ${ }^{14}$, who, after reviewing several papers, concluded that about 19\% of lactose maldigesters reported symptoms after ingestion of $240 \mathrm{ml}$ of milk. However, the proportion of maldigesting women reporting symptoms in our study is less than that reported by Rao et al., which was $73 \%{ }^{15}$, and Bayless ${ }^{16}$, who reported that $50 \%$ of adult maldigesters experienced symptoms after ingestion of $240 \mathrm{ml}$ of milk.

Limitations exist in this study. There was a loss to followup in the pregnant population. A proportion of this loss was due to biological causes (such as miscarriages and pre-term births). Those women who were unable to return for the late pregnancy testing due to early delivery were asked to return for the postpartum test. Sixteen of them did return and are included in the postpartum results. The remaining losses are due to the inherent difficulty in following a group of young, low-income women during this transitional life period.

In conclusion, this study indicates that the prevalence of lactose maldigestion in pregnant African-American women is similar to that in non-pregnant AfricanAmerican women and similar to previous prevalence reports in adult African-Americans. There was no change in lactose digestion noted during pregnancy in these women. However, there were fewer symptoms of lactose intolerance reported by lactose-maldigesting pregnant women. The prevalence of lactose maldigestion in pregnant African-American women, irrespective of pregnancy status or stage of pregnancy, parallels the prevalence of lactose maldigestion reported in adult African-Americans.

Lactose maldigestion, as measured by breath hydrogen response to $240 \mathrm{ml}$ of low-fat (1\%) milk, reinforces the IOM's concern with lactose digestion among pregnant African-American women. Healthcare providers instructing African-American women on the optimal dietary pattern during pregnancy need to be mindful of a high rate of lactose maldigestion. In this regard, Kingfisher and Millard ${ }^{17}$ reported that: 'Euroamerican staff tended to give advice that was biologically appropriate for them but not for many of their patients, a process reflecting biocentrism'. Whether this concern is valid remains to be answered by further study. Furthermore, health providers need to be aware that the presence or absence of symptoms may be reported unevenly by pregnant African-American women; and symptoms do not represent a reliable guide to lactose digestion. Less than $25 \%$ of pregnant lactose-maldigesting women reported any symptoms with $240 \mathrm{ml}$ of low-fat (1\%) milk. Symptoms may be reduced further when milk is consumed with other foods. Unanswered is the level of digestion and absorption of a range of nutrients in the milk consumed. Healthcare providers should discuss the ability to tolerate milk with the pregnant woman, and, where and when appropriate, should educate her as to other food options.

\section{Acknowledgements}

The authors acknowledge the invaluable editorial assistance of Ms Linda Kelly in the preparation of this manuscript. Support for this research was provided by The Maternal and Child Health Bureau of the US Department of Health and Human Services.

\section{References}

1 Institute of Medicine (US) Subcommittee on Nutritional Status and Weight Gain During Pregnancy. Nutrition During Pregnancy. Washington, DC: National Academy of Sciences, 1990.

2 Scrimshaw NS, Murray EB. The acceptability of milk and milk products in populations with a high prevalence of lactose intolerance. American Journal of Clinical Nutrition 1988; 48: 1079-159.

3 Rush D, Sloan N, Leighton J, Alvir JM, Horvitz DG, Seaver WB, et al. The National WIC Evaluation: evaluation of the Special Supplemental Food Program for Women, Infants, and Children. V. Longitudinal study of pregnant women. American Journal of Clinical Nutrition 1988; 48: 439-83.

4 Paige DM, Bayless TM, Graham GG. Pregnancy and lactose intolerance. American Journal of Clinical Nutrition 1973; 26: $238-40$.

5 Tandon RK, Goel U, Mukherjee SN, Pandey SC, Lal K. Lactose intolerance during pregnancy in different Indian communities. Indian Journal of Medical Research 1977; 66(1): 33-8. 
6 Villar J, Kestler E, Castillo P, Juarez A, Menendez R, Solomons NW. Improved lactose digestion during pregnancy: a case of physiologic adaptation? Obstetrics and Gynecology 1988; 71: 697-700.

7 Szilagyi A, Salomon R, Martin M, Fokeeff K, Seidman E. Lactose handling by women with lactose malabsorption is improved during pregnancy. Clinical and Investigative Medicine 1996; 19(6): 416-26.

8 Perman JA, Barr RB, Watkins JB. Sucrose malabsorption in children; non-invasive diagnosis by interval breath hydrogen determination. Journal of Pediatrics 1978; 93: 17-22.

9 Romagnuolo J, Schiller D, Bailey RJ. Using breath tests wisely in a gastroenterology practice: an evidence-based review of indications and pitfalls in interpretation. American Journal of Gastroenterology 2002; 97(5): 1113-26.

10 Bayless TM, Rosensweig NS. A racial difference in the incidence of lactase deficiency. Journal of the American Medical Association 1966; 197: 968-72.

11 Johnson AO, Semenya JG, Buchowski MS, Enwonwu CO, Scrimshaw NS. Adaptation of lactose maldigesters to continued milk intakes. American Journal of Clinical Nutrition 1993; 58: 879-81.

12 Parry E, Shields R, Turnbull AC. Transit time in the small intestine during pregnancy. Journal of Obstetrics and Gynaecology of the British Commonwealth 1970; 77: 900-1. 13 Labayen I, Forga L, Gonzalez A, Lenoir-Wijnkoop I, Nutr R, Martinez JA. Relationship between lactose digestion, gastrointestinal transit time and symptoms in lactose malabsorbers after dairy consumption. Alimentary Pharmacology $\&$ Therapeutics 2001; 15(4): 543-9.

14 Newcomer AD. Immediate symptomatic and long-term nutritional consequences of hypolactasia. In: Paige DM, Bayless TM, eds. Lactose Digestion: Clinical and Nutritional Implications. Baltimore, MD: Johns Hopkins University Press, 1981; 124-33.

15 Rao DR, Bello H, Warren AP, Brown GE. Prevalence of lactose maldigestion. Digestive Diseases and Sciences 1994; 39: 1519-24.

16 Bayless TM. Lactose malabsorption, milk intolerance, and symptom awareness in adults. In: Paige DM, Bayless TM, eds. Lactose Digestion: Clinical and Nutritional Implications. Baltimore, MD: Johns Hopkins University Press, 1981; 117-23.

17 Kingfisher CP, Millard AV. 'Milk makes me sick but my body needs it': conflict and contradiction in the establishment of authoritative knowledge. Medical Anthropology Quarterly 1998; 12(4): 447-66. 